\title{
Public health indicators for the EU: the joint action for ECHIM (European Community Health Indicators \& Monitoring)
}

Marieke Verschuuren ${ }^{1 *}$, Mika Gissler², Katri Kilpeläinen², Antti Tuomi-Nikula², Ari-Pekka Sihvonen², Jürgen Thelen ${ }^{3}$, Rita Gaidelyte ${ }^{4}$, Silvia Ghirini ${ }^{5}$, Nils Kirsch ${ }^{3}$, Remigijus Prochorskas ${ }^{4}$, Emanuele Scafato ${ }^{5}$, Pieter Kramers ${ }^{2}$ and Arpo Aromaa ${ }^{6}$

\begin{abstract}
Background: Public health policies aim to improve and maintain the health of citizens. Relevant data and indicators are needed for a health policy that is based on factual information. After 14 years of work (1998-2012), the multi-phase action on European Community Health Indicators (ECHI) has created a health monitoring and reporting system. It has generated EU added value by defining the ECHI shortlist with 88 common and comparable key health indicators for Europe.

Methods: In the 2009-2012 Joint Action for ECHIM project the ECHI shortlist was updated through consultation with Member State representatives. Guidelines for implementation of the ECHI Indicators at national level were developed and a pilot data collection was carried out.

Results: 67 of the ECHI Indicators are already part of regular international data collections and thus available for a majority of Member States, 14 are close to ready and 13 still need development work. By mid-2012 half of the countries have incorporated ECHI indicators in their national health information systems and the process is ongoing in the majority of the countries. Twenty-five countries were able to provide data in a Pilot Data Collection for 20 ECHI Indicators that were not yet (fully) available in the international databases.

Conclusions: The EU needs a permanent health monitoring and reporting system. The Joint Action for ECHIM has set an example for the implementation of a system that can develop and maintain the ECHI indicators, and promote and encourage the use of ECHI in health reporting and health policy making. The aim for sustainable public health monitoring is also supported by a Eurostat regulation on public health statistics requiring that health statistics shall be provided according to the ECHI methodology. Further efforts at DG SANCO and Eurostat are needed towards a permanent health monitoring system.
\end{abstract}

Keywords: Public health indicators, Public health monitoring, Public health reporting

\section{Background}

The major aim of public health policies is to improve and maintain the health of citizens and to reduce health inequalities. These policies have to be based on factual information drawn from relevant data and comparable indicators. Relevant health indicators enable correctly targeted policy measures and assessment of their impact.

\footnotetext{
* Correspondence: marieke.verschuuren@rivm.nl

${ }^{1}$ RIVM, National Institute for Public Health and the Environment, P.O. Box 1 $\mathrm{NL}$ - 3720, Bilthoven, BA, the Netherlands

Full list of author information is available at the end of the article
}

To reach this goal, health indicators have to be based on representative population-based health data and need to be comparable between points in time, countries and areas. Observed differences between countries can stimulate the improvement of national health systems. Therefore, a joint international health information system will help Member States to implement their public health monitoring and reporting system and thus enable them to carry out their public health responsibilities. For the EU, the implementation of relevant health indicators is an essential starting point for a common health

\section{Biomed Central}


monitoring and reporting system that is essential for supporting EU level public health policies.

The European Parliament has called for an effective health information system since the 1990s. The first step on the road to harmonisation was the launch of the European Commission's first Health Monitoring Programme in 1993. Under this Programme projects were financed to develop harmonized health indicators [1]. In 1996, the European Commission set up a working group to draft a proposal on how to organise health monitoring in the European Union [2]. The following year, the Amsterdam Treaty provided harmonised instructions on the public health responsibilities of the Member States [3].

The multi-phase action on European Community Health Indicators (ECHI) has been one of the core actions of the European Commission's Health Programmes for 14 years (1998-2012). Its main task was the development, maintenance and implementation of a set of general public health indicators, the ECHI shortlist. Several international indicator- and datasets already exist, both broad (e.g. Eurostat, WHO Health for All database, OECD health data) and topical (e.g. data collections by the EMCDDA and ECDC). Yet the ECHI shortlist provides added value because it has been developed as a concise yet comprehensive tool for policy support, rather than as a (data driven) database. The first two projects (ECHI 1998-2001 and ECHI-2 2002-2004) focused on the selection and definition of indicators, and established the first version of the ECHI shortlist in 2005 [4,5]. The $3^{\text {rd }}$ (ECHIM 2005-2008, M stands for Monitoring) [6] and the $4^{\text {th }}$ phase (Joint Action for ECHIM 2009-2012) shifted the focus towards the implementation of the ECHI indicators in the Member States and at EU level. A Joint Action is a specific financing mechanism that was newly introduced together with the EU Health Programme Together for Health in 2008. It involves a closed call from the Commission to the Member States to present a proposal, in contrast with normal project calls, which are open. In 2012, Prof. Aromaa described his personal reflections on the progress of the ECHI(M) projects in the broader perspective of past as well as necessary future developments [7].

The incorporation of ECHI Indicators into national health information systems is essential to ensure the continuous development and improvements in ECHI data availability, quality and comparability in the EU. During the $3^{\text {rd }}$ project phase $(2005-2008)$ a start has been made with this implementation of the ECHI Indicators at national level by, among other things, setting up a network of ECHI contact persons in the EU Member States and assessing availability of national data for ECHI Indicators in international databases [6]. The Joint Action for ECHIM continued this implementation work.
Next to enhancing implementation of ECHI Indicators at national level, its main objectives comprised updating and documenting the ECHI shortlist, and the assessment of availability and quality of data for ECHI Indicators that are not yet part of existing international data collections by means of a data collection pilot. In this article, we will describe the main results and experiences of the Joint Action for ECHIM.

\section{Methods}

There were five project partners in the Joint Action: the public health institutes of Finland (main partner), the Netherlands, Germany, Lithuania and Italy. Twenty-four Member States in total gave an official declaration of intent to participate in the Joint Action [8]. In practice, though, 36 countries (EU Member States, accession and candidate countries, and EFTA countries) participated. The Joint Action started on 1 January 2009 and ended on June $30^{\text {th }}$ 2012. Further methods applied are described according to the following three main objectives of the Joint Action:

\section{An updated and fully documented shortlist of ECHI indicators}

A new procedure for updating the shortlist was developed in 2010-2011, together with the Member State representatives of all countries participating in the Joint Action. Application of this new procedure resulted in the 2012 version of the ECHI shortlist. Clear criteria for additions or removals of indicators to/from the shortlist are at the core of the new updating procedure. Furthermore, the strong focus of the Joint Action on implementing the indicators is reflected in the criteria as well. The updating procedure has been described in detail in the final report of the Joint Action part II [9].

\section{Implementation of the ECHI shortlist indicators in participating EU countries}

The project partners created a model for the implementation plans for ECHI indicators, consisting of several elements (e.g. communication, data availability). Based on this model, guidelines for the Member States were developed at the beginning of the project. Progress of national implementation was monitored. The guidelines are described in detail in the Joint Action final report part I [10].

\section{Pilot data collection}

The existing international databases of Eurostat, the WHO Health for All database and OECD Health Data together with topic-specific international databases (e.g. ECDC and EMCDDA) are the recommended data source for 44 shortlist indicators. An ECHIM Pilot Data Collection was performed in 2010-2011 to obtain comparable 
Table 1 ECHI shortlist, 2012 version

\begin{tabular}{|c|c|c|c|c|c|}
\hline $\begin{array}{l}\text { ECHI shortlist } \\
\text { indicators }\end{array}$ & Data source & Status indicator & 25. Stroke & n.a. & $\begin{array}{l}\text { Work-in-progress } \\
\text { section }\end{array}$ \\
\hline 1. Population by sex/age & Eurostat & $\begin{array}{l}\text { Implementation } \\
\text { section }\end{array}$ & $\begin{array}{l}\text { 26. (A) Asthma, self- } \\
\text { reported prevalence }\end{array}$ & Eurostat (EHIS) & $\begin{array}{l}\text { Implementation } \\
\text { section }\end{array}$ \\
\hline 2. Birth rate, crude & Eurostat & $\begin{array}{l}\text { Implementation } \\
\text { section }\end{array}$ & $\begin{array}{l}\text { 26. (B) Asthma, register- } \\
\text { based prevalence }\end{array}$ & n.a. & $\begin{array}{l}\text { Work-in-progress } \\
\text { section }\end{array}$ \\
\hline $\begin{array}{l}\text { 3. Mother's age } \\
\text { distribution }\end{array}$ & Eurostat & $\begin{array}{l}\text { Implementation } \\
\text { section }\end{array}$ & $\begin{array}{l}\text { 27. }(\mathrm{A}) \mathrm{COPD} \text {, self- } \\
\text { reported prevalence }\end{array}$ & Eurostat (EHIS) & $\begin{array}{l}\text { Implementation } \\
\text { section }\end{array}$ \\
\hline 4. Total fertility rate & Eurostat & $\begin{array}{l}\text { Implementation } \\
\text { section }\end{array}$ & $\begin{array}{l}\text { 27. (B) COPD, register- } \\
\text { based prevalence }\end{array}$ & n.a. & $\begin{array}{l}\text { Work-in-progress } \\
\text { section }\end{array}$ \\
\hline 5. Population projections & Eurostat & $\begin{array}{l}\text { Implementation } \\
\text { section }\end{array}$ & 28. (Low) birth weight & WHO-HFA & $\begin{array}{l}\text { Implementation } \\
\text { section }\end{array}$ \\
\hline $\begin{array}{l}\text { 6. Population by } \\
\text { education }\end{array}$ & Eurostat (LFS) & $\begin{array}{l}\text { Implementation } \\
\text { section }\end{array}$ & $\begin{array}{l}\text { 29. (A) Injuries: home/ } \\
\text { leisure, self-reported }\end{array}$ & Eurostat (EHIS) & $\begin{array}{l}\text { Implementation } \\
\text { section }\end{array}$ \\
\hline 7. Population by & Eurostat (LFS) & Implementation & incidence & & \\
\hline occupation & & section & 29. (B) Injuries: home/ & $\mathrm{IDB}$ & Implementation \\
\hline 8. Total unemployment & Eurostat (LFS) & $\begin{array}{l}\text { Implementation } \\
\text { section }\end{array}$ & $\begin{array}{l}\text { leisure, register-based } \\
\text { incidence }\end{array}$ & & section \\
\hline $\begin{array}{l}\text { 9. Population below } \\
\text { poverty line and income } \\
\text { inequality }\end{array}$ & Eurostat (EU-SILC) & $\begin{array}{l}\text { Implementation } \\
\text { section }\end{array}$ & $\begin{array}{l}\text { 30. (A) Injuries: road } \\
\text { traffic, self-reported } \\
\text { incidence }\end{array}$ & Eurostat (EHIS) & $\begin{array}{l}\text { Implementation } \\
\text { section }\end{array}$ \\
\hline 10. Life expectancy & Eurostat & $\begin{array}{l}\text { Implementation } \\
\text { section }\end{array}$ & $\begin{array}{l}\text { 30. (B) Injuries: road traffic, } \\
\text { register-based incidence }\end{array}$ & UN ECE & $\begin{array}{l}\text { Implementation } \\
\text { section }\end{array}$ \\
\hline 11. Infant mortality & Eurostat & $\begin{array}{l}\text { Implementation } \\
\text { section }\end{array}$ & 31. Injuries: workplace & Eurostat (ESAW) & $\begin{array}{l}\text { Implementation } \\
\text { section }\end{array}$ \\
\hline 12. Perinatal mortality & WHO-HFA & $\begin{array}{l}\text { Implementation } \\
\text { section }\end{array}$ & 32. Suicide attempt & n.a. & $\begin{array}{l}\text { Development } \\
\text { section }\end{array}$ \\
\hline $\begin{array}{l}\text { 13. Disease-specific } \\
\text { mortality }\end{array}$ & $\begin{array}{l}\text { Eurostat (and CISID for } \\
\text { AIDS-related mortality) }\end{array}$ & $\begin{array}{l}\text { Implementation } \\
\text { section }\end{array}$ & 33. Self-perceived health & Eurostat (EU-SILC) & $\begin{array}{l}\text { Implementation } \\
\text { section }\end{array}$ \\
\hline 14. Drug-related deaths & EMCDDA & $\begin{array}{l}\text { Implementation } \\
\text { section }\end{array}$ & $\begin{array}{l}\text { 34. Self-reported chronic } \\
\text { morbidity }\end{array}$ & Eurostat (EU-SILC) & $\begin{array}{l}\text { Implementation } \\
\text { section }\end{array}$ \\
\hline $\begin{array}{l}\text { 15. Smoking-related } \\
\text { deaths }\end{array}$ & n.a. & $\begin{array}{l}\text { Work-in-progress } \\
\text { section }\end{array}$ & $\begin{array}{l}\text { 35. Long-term activity } \\
\text { limitations }\end{array}$ & Eurostat (EU-SILC) & $\begin{array}{l}\text { Implementation } \\
\text { section }\end{array}$ \\
\hline 16. Alcohol-related deaths & n.a. & $\begin{array}{l}\text { Work-in-progress } \\
\text { section }\end{array}$ & $\begin{array}{l}\text { 36. Physical and sensory } \\
\text { functional limitations }\end{array}$ & Eurostat (EHIS) & $\begin{array}{l}\text { Implementation } \\
\text { section }\end{array}$ \\
\hline $\begin{array}{l}\text { 17. Excess mortality by } \\
\text { extreme temperatures }\end{array}$ & n.a. & $\begin{array}{l}\text { Development } \\
\text { section }\end{array}$ & $\begin{array}{l}\text { 37. General } \\
\text { musculoskeletal pain }\end{array}$ & n.a. & $\begin{array}{l}\text { Development } \\
\text { section }\end{array}$ \\
\hline $\begin{array}{l}\text { 18. Selected } \\
\text { communicable diseases }\end{array}$ & ECDC & $\begin{array}{l}\text { Implementation } \\
\text { section }\end{array}$ & 38. Psychological distress & n.a. & $\begin{array}{l}\text { Development } \\
\text { section }\end{array}$ \\
\hline 19. HIV/AIDS & EURO-HIV/CISID & $\begin{array}{l}\text { Implementation } \\
\text { section }\end{array}$ & $\begin{array}{l}\text { 39. Psychological well- } \\
\text { being }\end{array}$ & n.a. & $\begin{array}{l}\text { Development } \\
\text { section }\end{array}$ \\
\hline 20. Cancer incidence & Globocan & $\begin{array}{l}\text { Implementation } \\
\text { section }\end{array}$ & $\begin{array}{l}\text { 40. Health expectancy: } \\
\text { Healthy Life Years (HLY) }\end{array}$ & Eurostat & $\begin{array}{l}\text { Implementation } \\
\text { section }\end{array}$ \\
\hline $\begin{array}{l}\text { 21. (A) Diabetes, self- } \\
\text { reported prevalence }\end{array}$ & Eurostat (EHIS) & $\begin{array}{l}\text { Implementation } \\
\text { section }\end{array}$ & $\begin{array}{l}\text { 41. Health expectancy, } \\
\text { others }\end{array}$ & EHEMU/EHLEIS project & $\begin{array}{l}\text { Work-in-progress } \\
\text { section }\end{array}$ \\
\hline $\begin{array}{l}\text { 21. (B) Diabetes, register- } \\
\text { based prevalence }\end{array}$ & n.a. & $\begin{array}{l}\text { Work-in-progress } \\
\text { section }\end{array}$ & 42. Body mass index & Eurostat (EHIS) & $\begin{array}{l}\text { Implementation } \\
\text { section }\end{array}$ \\
\hline 22. Dementia & n.a. & $\begin{array}{l}\text { Work-in-progress } \\
\text { section }\end{array}$ & 43. Blood pressure & Eurostat (EHIS) & $\begin{array}{l}\text { Implementation } \\
\text { section }\end{array}$ \\
\hline $\begin{array}{l}\text { 23. (A) Depression, self- } \\
\text { reported prevalence }\end{array}$ & Eurostat (EHIS) & $\begin{array}{l}\text { Implementation } \\
\text { section }\end{array}$ & 44. Regular smokers & Eurostat (EHIS) & $\begin{array}{l}\text { Implementation } \\
\text { section }\end{array}$ \\
\hline $\begin{array}{l}\text { 23. (B) Depression, } \\
\text { register-based prevalence }\end{array}$ & n.a. & $\begin{array}{l}\text { Work-in-progress } \\
\text { section }\end{array}$ & $\begin{array}{l}\text { 45. Pregnant women } \\
\text { smoking }\end{array}$ & n.a. & $\begin{array}{l}\text { Work-in-progress } \\
\text { section }\end{array}$ \\
\hline $\begin{array}{l}\text { 24. Acute Myocardial } \\
\text { Infarction }\end{array}$ & n.a. & $\begin{array}{l}\text { Work-in-progress } \\
\text { section }\end{array}$ & $\begin{array}{l}\text { 46. Total alcohol } \\
\text { consumption }\end{array}$ & WHO (GISAH) & $\begin{array}{l}\text { Implementation } \\
\text { section }\end{array}$ \\
\hline
\end{tabular}

Table 1 ECHI shortlist, 2012 version (Continued)

26. (A) Asthma, self-

26. (B) Asthma, registerprevalence

27. (A) COPD, self-

27. (B) COPD, registerbased prevalence

29. (A) Injuries: home/ incidence register-based incidence 
Table 1 ECHI shortlist, 2012 version (Continued)

\begin{tabular}{|c|c|c|}
\hline $\begin{array}{l}\text { 47. Hazardous alcohol } \\
\text { consumption }\end{array}$ & Eurostat (EHIS) & $\begin{array}{l}\text { Implementation } \\
\text { section }\end{array}$ \\
\hline 48. Use of illicit drugs & EMCDDA & $\begin{array}{l}\text { Implementation } \\
\text { section }\end{array}$ \\
\hline 49. Consumption of fruit & Eurostat (EHIS) & $\begin{array}{l}\text { Implementation } \\
\text { section }\end{array}$ \\
\hline $\begin{array}{l}\text { 50. Consumption of } \\
\text { vegetables }\end{array}$ & Eurostat (EHIS) & $\begin{array}{l}\text { Implementation } \\
\text { section }\end{array}$ \\
\hline 51. Breastfeeding & WHO-HFA & $\begin{array}{l}\text { Work-in-progress } \\
\text { section }\end{array}$ \\
\hline 52. Physical activity & Eurostat (EHIS) & $\begin{array}{l}\text { Implementation } \\
\text { section }\end{array}$ \\
\hline $\begin{array}{l}\text { 53. Work-related health } \\
\text { risks }\end{array}$ & EUROFOUND & $\begin{array}{l}\text { Implementation } \\
\text { section }\end{array}$ \\
\hline 54. Social support & Eurostat (EHIS) & $\begin{array}{l}\text { Implementation } \\
\text { section }\end{array}$ \\
\hline $\begin{array}{l}\text { 55. PM10 (particulate } \\
\text { matter) exposure }\end{array}$ & Eurostat & $\begin{array}{l}\text { Implementation } \\
\text { section }\end{array}$ \\
\hline $\begin{array}{l}\text { 56. Vaccination coverage } \\
\text { in children }\end{array}$ & WHO-HFA & $\begin{array}{l}\text { Implementation } \\
\text { section }\end{array}$ \\
\hline $\begin{array}{l}57 . \text { Influenza vaccination } \\
\text { rate in elderly }\end{array}$ & Eurostat (EHIS) & $\begin{array}{l}\text { Implementation } \\
\text { section }\end{array}$ \\
\hline $\begin{array}{l}\text { 58. Breast cancer } \\
\text { screening }\end{array}$ & Eurostat (EHIS) & $\begin{array}{l}\text { Implementation } \\
\text { section }\end{array}$ \\
\hline $\begin{array}{l}\text { 59. Cervical cancer } \\
\text { screening }\end{array}$ & Eurostat (EHIS) & $\begin{array}{l}\text { Implementation } \\
\text { section }\end{array}$ \\
\hline $\begin{array}{l}\text { 60. Colon cancer } \\
\text { screening }\end{array}$ & Eurostat (EHIS) & $\begin{array}{l}\text { Implementation } \\
\text { section }\end{array}$ \\
\hline $\begin{array}{l}\text { 61. Timing of first } \\
\text { antenatal visits among } \\
\text { pregnant women }\end{array}$ & n.a. & $\begin{array}{l}\text { Work-in-progress } \\
\text { section }\end{array}$ \\
\hline 62. Hospital beds & Eurostat & $\begin{array}{l}\text { Implementation } \\
\text { section }\end{array}$ \\
\hline 63. Practising physicians & Eurostat & $\begin{array}{l}\text { Implementation } \\
\text { section }\end{array}$ \\
\hline 64. Practising nurses & Eurostat & $\begin{array}{l}\text { Implementation } \\
\text { section }\end{array}$ \\
\hline $\begin{array}{l}\text { 65. Mobility of } \\
\text { professionals }\end{array}$ & n.a. & $\begin{array}{l}\text { Development } \\
\text { section }\end{array}$ \\
\hline $\begin{array}{l}\text { 66. Medical technologies: } \\
\text { MRI units and CT scans }\end{array}$ & Eurostat & $\begin{array}{l}\text { Implementation } \\
\text { section }\end{array}$ \\
\hline $\begin{array}{l}\text { 67. Hospital in-patient } \\
\text { discharges, limited } \\
\text { diagnoses }\end{array}$ & Eurostat & $\begin{array}{l}\text { Implementation } \\
\text { section }\end{array}$ \\
\hline $\begin{array}{l}\text { 68. Hospital day-cases, } \\
\text { limited diagnoses }\end{array}$ & Eurostat & $\begin{array}{l}\text { Implementation } \\
\text { section }\end{array}$ \\
\hline $\begin{array}{l}\text { 69. Hospital day-cases as } \\
\text { percentage of total } \\
\text { patient population (in- } \\
\text { patients \& day-cases), } \\
\text { selected diagnoses }\end{array}$ & $\begin{array}{l}\text { Eurostat (necessary } \\
\text { discharge data available } \\
\text { but ratio is not centrally } \\
\text { computed yet) }\end{array}$ & $\begin{array}{l}\text { Implementation } \\
\text { section }\end{array}$ \\
\hline $\begin{array}{l}\text { 70. Average length of } \\
\text { stay (ALOS), limited } \\
\text { diagnoses }\end{array}$ & Eurostat & $\begin{array}{l}\text { Implementation } \\
\text { section }\end{array}$ \\
\hline $\begin{array}{l}\text { 71. General practitioner } \\
\text { (GP) utilisation }\end{array}$ & Eurostat (EHIS) & $\begin{array}{l}\text { Implementation } \\
\text { section }\end{array}$ \\
\hline
\end{tabular}

Table 1 ECHI shortlist, 2012 version (Continued)

\begin{tabular}{|c|c|c|}
\hline $\begin{array}{l}\text { 72. Selected outpatient } \\
\text { visits }\end{array}$ & Eurostat (EHIS) & $\begin{array}{l}\text { Implementation } \\
\text { section }\end{array}$ \\
\hline 73. Selected Surgeries & Eurostat & $\begin{array}{l}\text { Implementation } \\
\text { section }\end{array}$ \\
\hline $\begin{array}{l}\text { 74. Medicine use, selected } \\
\text { groups }\end{array}$ & Eurostat (EHIS) & $\begin{array}{l}\text { Implementation } \\
\text { section }\end{array}$ \\
\hline 75. Patient mobility & $\begin{array}{l}\text { Eurostat is regularly } \\
\text { collecting data on } \\
\text { patient mobility but is } \\
\text { not yet publishing } \\
\text { these. }\end{array}$ & $\begin{array}{l}\text { Work-in-progress } \\
\text { section }\end{array}$ \\
\hline 76. Insurance coverage & OECD & $\begin{array}{l}\text { Implementation } \\
\text { section }\end{array}$ \\
\hline $\begin{array}{l}\text { 77. Expenditures on } \\
\text { health }\end{array}$ & Eurostat & $\begin{array}{l}\text { Implementation } \\
\text { section }\end{array}$ \\
\hline 78. Survival rates cancer & EUROCARE & $\begin{array}{l}\text { Implementation } \\
\text { section }\end{array}$ \\
\hline $\begin{array}{l}\text { 79. } 30 \text {-day in-hospital } \\
\text { case-fatality Acute } \\
\text { Myocardial Infarction and } \\
\text { stroke }\end{array}$ & OECD & $\begin{array}{l}\text { Implementation } \\
\text { section }\end{array}$ \\
\hline $\begin{array}{l}\text { 80. Equity of access to } \\
\text { health care services }\end{array}$ & Eurostat (EU-SILC) & $\begin{array}{l}\text { Implementation } \\
\text { section }\end{array}$ \\
\hline $\begin{array}{l}\text { 81. Waiting times for } \\
\text { elective surgeries }\end{array}$ & n.a. & $\begin{array}{l}\text { Development } \\
\text { section }\end{array}$ \\
\hline $\begin{array}{l}\text { 82. Surgical wound } \\
\text { infections }\end{array}$ & n.a. & $\begin{array}{l}\text { Development } \\
\text { section }\end{array}$ \\
\hline $\begin{array}{l}\text { 83. Cancer treatment } \\
\text { delay }\end{array}$ & n.a. & $\begin{array}{l}\text { Development } \\
\text { section }\end{array}$ \\
\hline 84. Diabetes control & n.a. & $\begin{array}{l}\text { Development } \\
\text { section }\end{array}$ \\
\hline $\begin{array}{l}\text { 85. Policies on ETS } \\
\text { exposure (Environmental } \\
\text { Tobacco Smoke) }\end{array}$ & $\begin{array}{l}\text { WHO-Euro tobacco } \\
\text { control (computation of } \\
\text { indicator not done } \\
\text { centrally yet) }\end{array}$ & $\begin{array}{l}\text { Implementation } \\
\text { section }\end{array}$ \\
\hline $\begin{array}{l}\text { 86. Policies on healthy } \\
\text { nutrition }\end{array}$ & n.a. & $\begin{array}{l}\text { Development } \\
\text { section }\end{array}$ \\
\hline $\begin{array}{l}\text { 87. Policies and practices } \\
\text { on healthy lifestyles }\end{array}$ & n.a. & $\begin{array}{l}\text { Development } \\
\text { section }\end{array}$ \\
\hline $\begin{array}{l}\text { 88. Integrated } \\
\text { programmes in settings, } \\
\text { including workplace, } \\
\text { schools, hospital }\end{array}$ & n.a. & $\begin{array}{l}\text { Development } \\
\text { section }\end{array}$ \\
\hline
\end{tabular}

data for $20 \mathrm{ECHI}$ shortlist indicators that were unavailable or incomparable in these international databases. For many of these 20 indicators the European Health Interview Survey (EHIS) is the preferred data source. Therefore, ECHI-conform data were obtained from Eurostat for Member States for which EHIS micro-datasets were available from EHIS wave I, which was carried out in the period 2006-2010 [11]. These were complemented with data from national Health Interview Surveys from Member States that had not participated in EHIS wave I. Out of the 36 countries participating in the Joint Action, in 34 
suitable contact persons were identified for receiving a Pilot Data Collection questionnaire.

\section{Results}

\section{An updated and fully documented shortlist of ECHI} indicators

After introducing the ECHI shortlist in 2005 [5], the indicator metadata for all 88 indicators in the ECHI shortlist has been documented and continuously improved, and the ECHI shortlist has been updated in 2008 [6] and 2012 [9]. The 2008 version comprised an implementation and a development section. During the Joint Action, however, a more precise definition of the indicators and a stronger focus on implementation led to splitting the development section into a work-in-progress section in addition to the development section. Therefore, the 2012 version of the ECHI shortlist is divided into three rather than two sections.

The 67 ECHI Indicators in the implementation section are already part of regular international data collections, and data are available for a majority of Member States, and thus ready for implementation. The 14 ECHI indicators in the work-in-progress section are almost ready to be included in regular international data collections. In most cases, however, no concrete plans exist for this at present. The remaining $13 \mathrm{ECHI}$ indicators in the development section contain topics that are needed for policy support, but that are not ready yet for incorporation in international regular data collections and for implementation. Please note that there are 88 indicators in the ECHI shortlist. However, six of these have two different operationalizations: one based on self-reported data and one based on administrative or register-based data. Both operationalizations have been assessed separately here, resulting in a total of $67+14+13=94$ indicators. An overview of the 2012 version of the ECHI shortlist is presented in Table 1.

A report documenting the indicators and the work performed has been published [9]. This 'cookbook' for the ECHI shortlist indicators is aimed at serving persons working with the indicators, computing them, or making them available. In addition, the European Commission made an up-to-date presentation of data and metadata for multiple indicators in its HEIDI tool [12].

\section{Implementation of the ECHI shortlist indicators in participating EU countries}

During the Joint Action for ECHIM, ten countries already started incorporating ECHI into their national databases: Austria, Czech Republic, Estonia, Germany, Greece, Italy, Lithuania, Latvia, the Netherlands and Spain. Some countries have created national offline data presentations of ECHI indicators, for example using national customised versions of the WHO Data Presentation
System (DPS), as has been done in Lithuania [13]. In addition, a number of other countries have started using the ECHI framework in their health reports, e.g. the Netherlands [14] and France [15]. A number of countries in June 2012 reported having concrete plans to incorporate $\mathrm{ECHI}$ in the national indicator and/or data presentation system (e.g. Malta, Finland, Ireland, and Norway). This shows for example from stating in future action plans of National Public Health Institutes or strategies of the Ministries of Health that there is an intention to use ECHI indicators in national health monitoring and reporting systems.

\section{Pilot data collection}

In total 25 countries provided data in the pilot (see Figure 1). No pilot data at all were received from nine countries (Bulgaria, Greece, Luxembourg, Portugal, Sweden, Slovenia, Slovakia, Hungary and Turkey). However, none of the participating countries was able to provide all requested indicators and breakdowns. Furthermore, conceptual and methodological differences among national HIS data hampered valid mutual comparisons, as well as clear-cut comparisons with the EHIS-based data. A separate final report of the Joint Action documenting in detail the ECHIM Pilot Data Collection, data received and the analyses has been published in 2013 (part III) [16].

\section{Discussion}

The European Parliament has highlighted the need for a public health information system. High quality health information serves the EU and Member States by helping to direct health, welfare and other policies and planning toward meeting peoples' health needs. Comparative health information is of great practical use not only for policy makers and planners, but also for health professionals, teachers, students, researchers, journalists, and the general population.

There is ample evidence from many European countries about the usefulness of policy relevant and comparable health information. The Joint Action for ECHIM contributes directly to the 2008-2013 Health Strategy of the European Commission [17].

DG SANCO has improved dissemination of ECHI indicators at the EU level by developing the HEIDI tool [12]. Another positive development is the Eurostat regulation on statistics on public health and health and safety at work [18], including the implementing regulation on EHIS [19], which refer to the ECHI shortlist. These will support the improved comparability of data across countries. Furthermore, increasing cooperation between the European Commission, WHO Regional Office for Europe and OECD, for example in the field of health expenditures statistics, is resulting in better data quality 


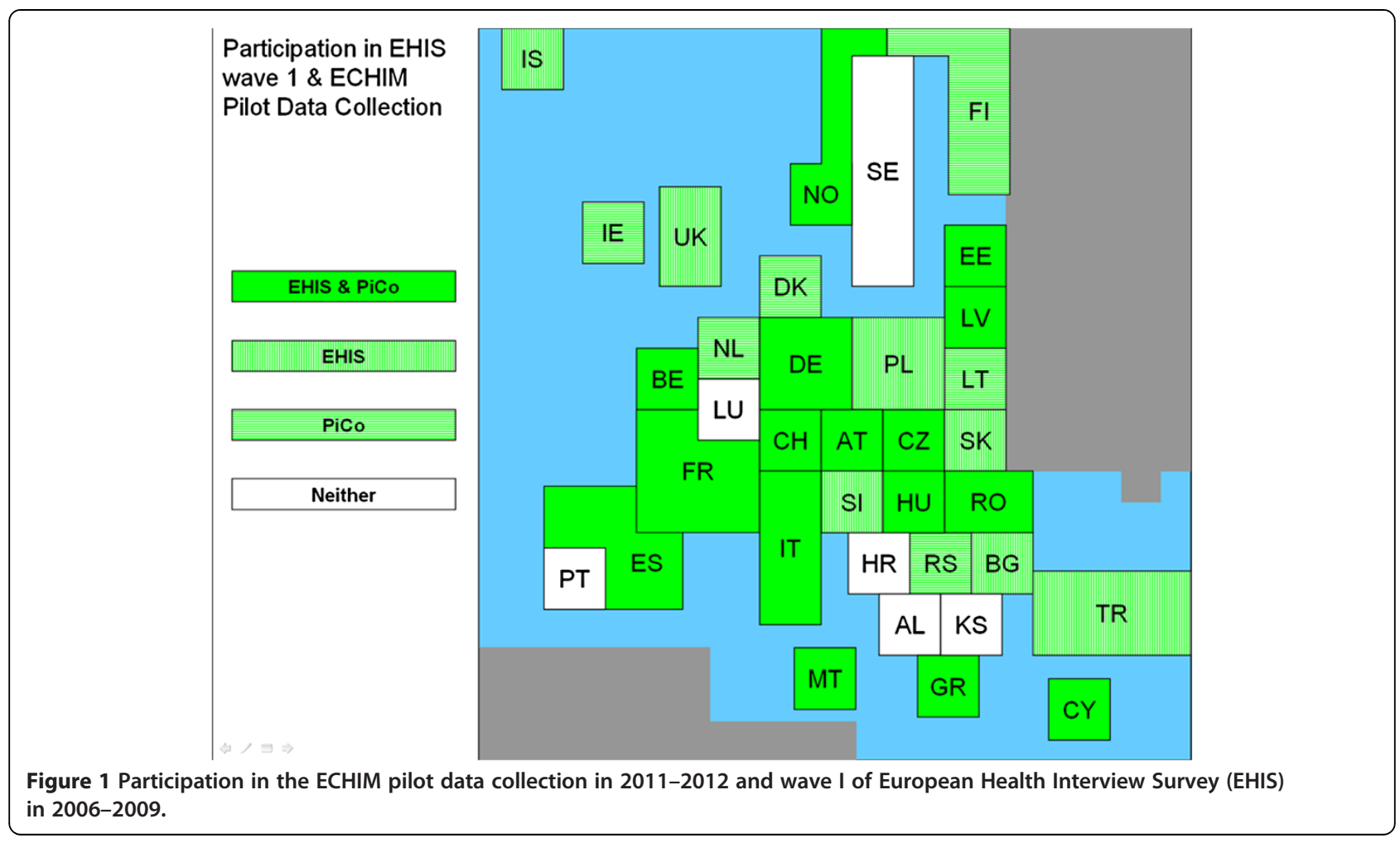

and comparability and less administrative burden for the Member States. Finally, the Member States have been involved in all the development steps of ECHI/ECHIM, and they increasingly use the ECHI shortlist for their own health information strategies. Nevertheless, despite years of work, substantial efforts are still needed at both Commission and national level to build-up and maintain a sustainable, high quality and easy-access evidence base for policy makers and other professionals. An important reason for this is that EU activities related to the development of elements of a health information system (e.g. indicators, standards for data collection, reporting mechanisms) are usually funded on an ad hoc project basis, resulting in a scattered and unsustainable situation.

Therefore it may not come as a surprise that after the ending of the Joint Action for ECHIM in June 2012, no follow up structure was put in place by the Commission. The Commission is performing a formal evaluation of the use and usefulness of ECHI in/for the Member States in 2013 and thereafter a decision about ECHI's future will be taken. The success and future of ECHI, however, depends on the ability of a central EU organization to organise and implement the collection and use of ECHI data at EU level in health monitoring and reporting. A health information system like ECHI needs constant maintenance, e.g. in the form of metadata compilation and indicator improvement. In addition, the countries need to be able to provide required data in a timely way and with sufficient quality, preferably through international data collection systems. The Member States have a major role to play, since they have to implement the ECHI Indicators in practice.

The Joint Action faced several obstacles that call for improvements. Most importantly, a stronger leadership at EU level and enhanced funding, dedicated personnel, and commitment at national level are needed in order to speed up the action. In the future, the logical and viable perspective would be to integrate the work on ECHI-defined data both with the collection of national health data and with the delivery of data to other international databases, such as those of Eurostat, WHO and OECD. Such a solution would be a coherent investment of resources, aimed at constantly improving the availability and cross-national comparability of health data.

Finally, health information and knowledge should be emphasized in the forthcoming Health for Growth Programme for 2014-2020. The Joint Action for ECHIM has prepared good documentation and methods to support the national and international work on health indicators, health monitoring and health reporting.

\section{Conclusions}

The ECHI Indicators were developed as the core of a European health information and reporting system, and have proven their usefulness and added value in practice. Sustained efforts at EU and national level are needed to keep the system functional and up to date. 


\section{Abbreviations}

DG SANCO: European commission's directorate general for health \& consumers; DPS: Data presentation system; ECDC: European centre of disease prevention and control; ECHI: European community health indicators; ECHIM: European community health indicators and monitoring; EHIS: European health interview survey; EFTA: European free trade association; EMCDDA: European monitoring centre for drugs and drug addiction; EU: European union; Eurostat: Statistical office of the European union; HEIDI: Health in Europe: information and data interface; OECD: Organisation for Economic Co-operation and Development; WHO: World Health Organisation

\section{Competing interests}

The authors declared that they have no competing interest

\section{Authors' contributions}

All authors participated in the design of the study and data preparation. MV MG and KK drafted the paper with the help of AT-N and A-PS. All authors reviewed and commented on the manuscript before submission and approved the final version.

\section{Acknowledgements}

Joint Action ECHIM has received funding from the European Commission / Directorate General for Health and Consumers (Grant agreement number 200823 91), as well as from the national authorities of the five partner countries in the Joint Action for ECHIM. In the Netherlands, the Netherlands Organisation for Health Research and Development (ZonMw) also provided part of the co-funding, next to the $\mathrm{MoH}$. The views expressed here are those of the authors and they do not represent the Commission's official position. This article is based on the Final Reports of the Joint Action for ECHI $[8,9,16]$.

\section{Author details}

'RIVM, National Institute for Public Health and the Environment, P.O. Box 1 $\mathrm{NL}$ - 3720, Bilthoven, BA, the Netherlands. ${ }^{2} \mathrm{THL}$, National Institute for Health and Welfare, P.O. Box 30FI - 00270, Helsinki, Finland. ${ }^{3}$ Robert Koch Institute, P.O. Box 6502 61D-13302, Berlin, Germany. ${ }^{4}$ Center of Health Information, Institute of Hygiene, Didzioji 22, LT - 01128, Vilnius, Lithuania. ${ }^{5}$ ISS, Istituto Superiore di Sanità, Viale Regina Elena, 299, IT - 00161, Rome, Italy. ${ }^{6}$ Ratakatu 1a, Fl-00120, Helsinki, Finland.

Received: 23 February 2013 Accepted: 20 April 2013

Published: 30 May 2013

\section{References}

1. Commission of the European Communities: Commission communication on the framework for action in the field of public health. Report No.: COM (93) 559. Brussels: Commission of the European Communities; 1993.

2. Aromaa A: Health monitoring for the European Union. A report of an expert group set up by the commission to advise on the organisation of health monitoring. Helsinki: National Public Health Institute; 2000

3. European Communities: Treaty of Amsterdam: Amending the treaty on European Union, The treaties establishing the European communities and certain related acts. Official publication of the European Communities. Report No.: C 340. Luxembourg: Office for Official Publications of the European Communities; 1997.

4. Kramers $P$, the ECHI team: Context, selection, definition. In Final report by the ECHI project phase II. Edited by Public health indicators for the European Union. Bilthoven: Centre for Public Health Forecasting, National Institute for Public Health and the Environment; 2005.

5. Kramers P: The ECHI project, Health indicators for the European Community. Eur J Public Health 2003, 13:101-106.

6. Kilpeläinen K, Aromaa A, the ECHIM Core Group: Development and initial implementation. In Final report of the ECHIM project. Edited by European health indicators. Helsinki: Helsinki University Press; 2008.

7. Aromaa A: Implementation of joint health indicators in Europe - Joint Action for ECHIM, Arpo Aromaa on behalf of the ECHIM core group. Arch Public Health 2012, 70:22. doi:10.1186/0778-7367-70-22.

8. Verschuuren M, Kramers P, Gudfinnsdottir G, Aromaa A: Providing a solid evidence base for policy makers: ECHI initiative. EuroHealth 2010, 16:3.
9. Verschuuren M, Achterberg PW, Gijsen R, Harbers MM, Vijge E, Wilk EA Van D, Kramers PGN: ECHI indicator development and documentation. Joint Action for ECHIM Final Report Part II. Bilthoven: ECHIM project partners; 2012.

10. Tuomi-Nikula A, Gissler M, Sihvonen A-P, Kilpeläinen K, and the ECHIM Core Group: Implementation of European Health Indicators - first years. Helsinki: National Institute for Health and Welfare (THL); 2012

11. DG SANCO website: Data collection - Tools - Improving health reporting mechanisms. [http://ec.europa.eu/health/data_collection/tools/mechanisms/ index_en.htm\#fragment0.

12. European Commission: HEIDI Data Tool. http://ec.europa.eu/health/ indicators/indicators/index_en.htm.

13. Lithuanian customised version of the WHO Data Presentation System (DPS). http://sic.hi.lt/html/en//hic.htm

14. Harbers MM, Van der Wilk EA, Kramers PGN, Kuunders MMAP, Verschuuren M, Eliyahu H, Achterberg P: Dare to Compare! Benchmarking Dutch health with the European Community Health Indicators (ECHI). RIVM report number 270051011. Houten: Bohn Stafleu Van Loghum; 2008.

15. La santé en France et en Europe: Convergences et contrastes. Paris: Direction de l'information légale et administrative \& Haut Conseil de la Santé Publique; 2012.

16. Thelen J, Kirsch NH, Finger J: Joint Action for ECHIM: Final Report Part III ECHIM Pilot Data Collection, Analyses and Dissemination. Berlin, Germany: Robert Koch Institute; 2013

17. Commission of the European Communities: White paper: Together for Health: A Strategic Approach for the EU 2008-2013. Brussels: Commission of the European Communities; 2007.

18. Off J Eur Union Regulation (EC) No 1338/2008 of the European Parliament and of the Council of December 2008 on Community statistics on public health and safety at work. 354/70:dd31-12-2008.

19. J Eur Union L Commission Regulation (EU) No 141/2013 of 19 February 2013 implementing Regulation (EC) No 1338/2008 of the European Parliament and of the Council on Community statistics on public health and health and safety at work, as regards statistics based on the European Health Interview Survey (EHIS). 47/20:dd20.2.2013.

doi:10.1186/0778-7367-71-12

Cite this article as: Verschuuren et al:: Public health indicators for the EU: the joint action for ECHIM (European Community Health Indicators \& Monitoring). Archives of Public Health 2013 71:12.

\section{Submit your next manuscript to BioMed Central and take full advantage of:}

- Convenient online submission

- Thorough peer review

- No space constraints or color figure charges

- Immediate publication on acceptance

- Inclusion in PubMed, CAS, Scopus and Google Scholar

- Research which is freely available for redistribution

Submit your manuscript at www.biomedcentral.com/submit
C Biomed Central 